\title{
Innovación Política para una Nueva Realidad Social
}

\section{Innovative Policies for a New Social Reality}

\author{
Teresa Llorens Carbonell \\ Área de Igualdad y Ciudadanía de la Diputación de Barcelona
}

\begin{abstract}
Resumen. Cambios sociales como la llegada de inmigración piden que las administraciones públicas reaccionemos y diseñemos nuevas políticas que den respuestas a las necesidades de la ciudadanía. Políticas proactivas, que den elementos a las personas para afrontar este cambio social con tranquilidad, desde el conocimiento; políticas dirigidas no sólo al colectivo inmigrante, sinó al conjunto de personas que viven en nuestros pueblos y ciudades. Desde el Área de Igualdad y Ciudadanía de la Diputación de Barcelona, apostamos por las políticas de ciudadanía. Aquellas que situan en el centro de la acción pública a todas las personas, con el objetivo de trabajar para una mejor convivencia y cohesión social. Los Ayuntamientos, como administración de proximidad, deben liderar esta nueva acción y hacer frente a los retos que les presenta.

Palabras clave: migración / inmigración, ciudadanía, políticas públicas, derechos, deberes, Ayuntamiento, administración local, acogida, integración, igualdad, oportunidades.
\end{abstract}

\begin{abstract}
Summary
Social changes, such as the arrival of immigration, demand that public authorities react and design new policies that meet the needs of citizens. Proactive policies, giving people the tools to face this social change with peace of mind, from knowledge; policies aimed not just at immigrants, but at everyone living in our towns and cities. At the Department of Equality and Citizenship of the Diputació de Barcelona, we are committed to citizenship policies. Those that put everyone at the centre of public action, with the objective of working towards better living together and social cohesion. Town Councils, as local administration, must lead this action and face the challenges that it presents.

Key words: migration / immigration, citizenship, public policies, rights, duties, town council, administration, local, settlement, integration, equality, opportunities.
\end{abstract}

Los procesos migratorios y el incremento de la diversidad son, sin duda, algunos de los principales factores de cambio social. Se trata de procesos globales que tienen su principal expresión en la localidad de los municipios. Por ello, a las administraciones públicas se nos plantea la necesidad de actuar coordinadamente ante esta situación. Y desde el Área de Igualdad y Ciudadanía de la Diputación de Barcelona tenemos el convencimiento de que esta

La correspondencia se le puede enviar a la autora: Diputació de Barcelona, Área d'Igualitat i Ciutadania. Recinte Maternitat, Pavelló Mewstral. Travessera de les Corts, 131-159, $2^{\mathrm{a}}$ planta, 08008 Barcelona. E-mail: llorensct@diba.cat respuesta debe llegar desde la transversalidad de las políticas de ciudadanía.

En los últimos años, una nueva realidad social, la inmigración, está produciendo un cambio en la centralidad de la agenda de los gobiernos y plantea nuevos retos para el conjunto de las políticas públicas.

La globalización económica, las migraciones, la diversidad o la revolución tecnológica son algunos de los factores que se producen con rapidez y operan en la sociedad actual, son ejemplos de los conflictos que la governabilidad tiene que abordar desde nuevas formas de actuación y de decisión 
política. Se trata de factores, y de cambios, que tienen un origen global pero consecuencias locales, como pueden ser la percepción de la pérdida de la identidad simbólica del barrio por la llegada de nueva población o la distinta concepción que unos y otros tenemos del uso del espacio público, de la calle y las plazas.

En Cataluña estamos acostumbrados a recibir grandes flujos de inmigración desde siglos atrás, puesto que nuestra historia está estrechamente relacionada con la llegada de personas que han escogido Cataluña para iniciar un nuevo proyecto vital. Y con sus aportaciones han transformado y enriquecido nuestra identidad.

Pero en pocos años hemos obtenido cifras que en otros países han tardado décadas. Desde finales de los noventa, la llegada de población extrangera se ha acelerado de manera intensa. A modo de ejemplo, en Cataluña, en el año 2000 teníamos un 2,9\% de población inmigrada, y en el 2007 ya habíamos llegado al 13,5\%. Ello representa que del 2000 al 2007 el incremento de la población de nacionalidad extrangera en Cataluña ha sido del $436 \%$.

Es evidente, pues, que los poderes públicos, especialmente los gobiernos locales, se han visto con la obligación de afrontar esta nueva realidad, y de definir políticas públicas que les permitan afrontar este cambio social. Dibujando acciones que favorezcan la integración y la convivencia ciudadana, acciones que aseguren la condición de ciudadanía de todas las personas, que garantizen el respeto a los derechos y deberes, la igualdad de oportunidades y el acceso a los servicios y recursos sociales normalizados.

\section{Retos de los Ayuntamientos}

Fruto de nuestra experiencia y de nuestro trabajo con los Ayuntamientos, hemos observado que las administraciones locales han tenido que hacer frente a cuatro grandes retos: gestionar la recepción y acogida de las personas recién llegadas, garantizar la calidad de vida de la ciudadanía, garantizar la cantidad y la calidad de los servicios públicos, y gestionar y garantizar la integración y la convivencia ciudadana.

\section{Estrategias de recepción y acogida}

En estos primeros años de gestión de la inmigración, los Ayuntamientos, como institución más próxima a la ciudadanía, han dedicado buena parte de los programas municipales a las estrategias de recepción y acogida. Éste ha sido el primer reto.

Porque cuando una persona llega a nuestro país, es muy probable que desconozca algunos aspectos básicos relacionados con la cultura, el funcionamiento de las instituciones y de los servicios públicos, o aspectos de la vida cotidiana como por ejemplo dónde tiene que ir cuando se pone enferma.

Así pues, gestionar con políticas anticipativas estos primeros momentos de asentamiento de la población inmigrante es decisivo para asegurar la cohesión y la convivencia ciudadana, y minimizar procesos de segregación, marginación o conflicto, causados por las dificultades de instalación, la falta de información y el desconocimiento del nuevo entorno.

En el proceso de asentamiento se pueden distingir dos etapas básicas: la llegada y la instalación.

La llegada supone un primer contacto con la ciudad y el momento en que, por norma general, pueden aparecer dificultades en relación con la información sobre los servicios básicos, los trámites de documentación o las necesidades sociales en casos de urgencia social, entre otros. En esta fase, podemos encontrar población transeúnte, que vive en la ciudad durante un período corto de tiempo, u otra que, contrariamente, inicia el asentamiento en la ciudad procedente directamente de su país de origen, o que ha vivido temporadas en otros países europeos o en otras zonas del Estado español.

La instalación definitiva en una ciudad normalmente se decide cuando hay una cierta estabilidad laboral, se dispone de un lugar para residir y se ha estructurado una mínima red social de referencia. Por lo general, estos procesos van acompañados de reagrupamientos familiares. En estos momentos se hace imprescindible tener garantizado el acceso a los servicios públicos básicos (sanitarios, educativos, sociales, culturales...), así como disponer de la suficiente habilidad social para utilizarlos con la mayor autonomía posible.

En este sentido, consideramos que la fase de 
acogida se completa cuando el recién llegado se relaciona con autonomía con su entorno social y laboral más cercano. Por eso, "Las actuaciones orientadas a la gestión de la recepción y acogida de inmigrantes tienen que ser, necesariamente, transitorias. Tienen que facilitar la incorporación normalizada de la población inmigrante en la sociedad de destinación, momento en que se considera que el proceso de recepción o acogida finaliza" (Diputación de Barcelona, 2004). tos que facilitan el conocimiento del entorno a los recién llegados, y al mismo tiempo establecen una actuación coordinada de todas las administraciones públicas y entidades que operan en el ámbito de la acogida de la inmigración en un determinado territorio.

Es así como los planes locales de acogida aportan un valor añadido de innovación a la acción política e institucional de los Ayuntamientos, reforzando su rol de administración de proximidad, promoviendo

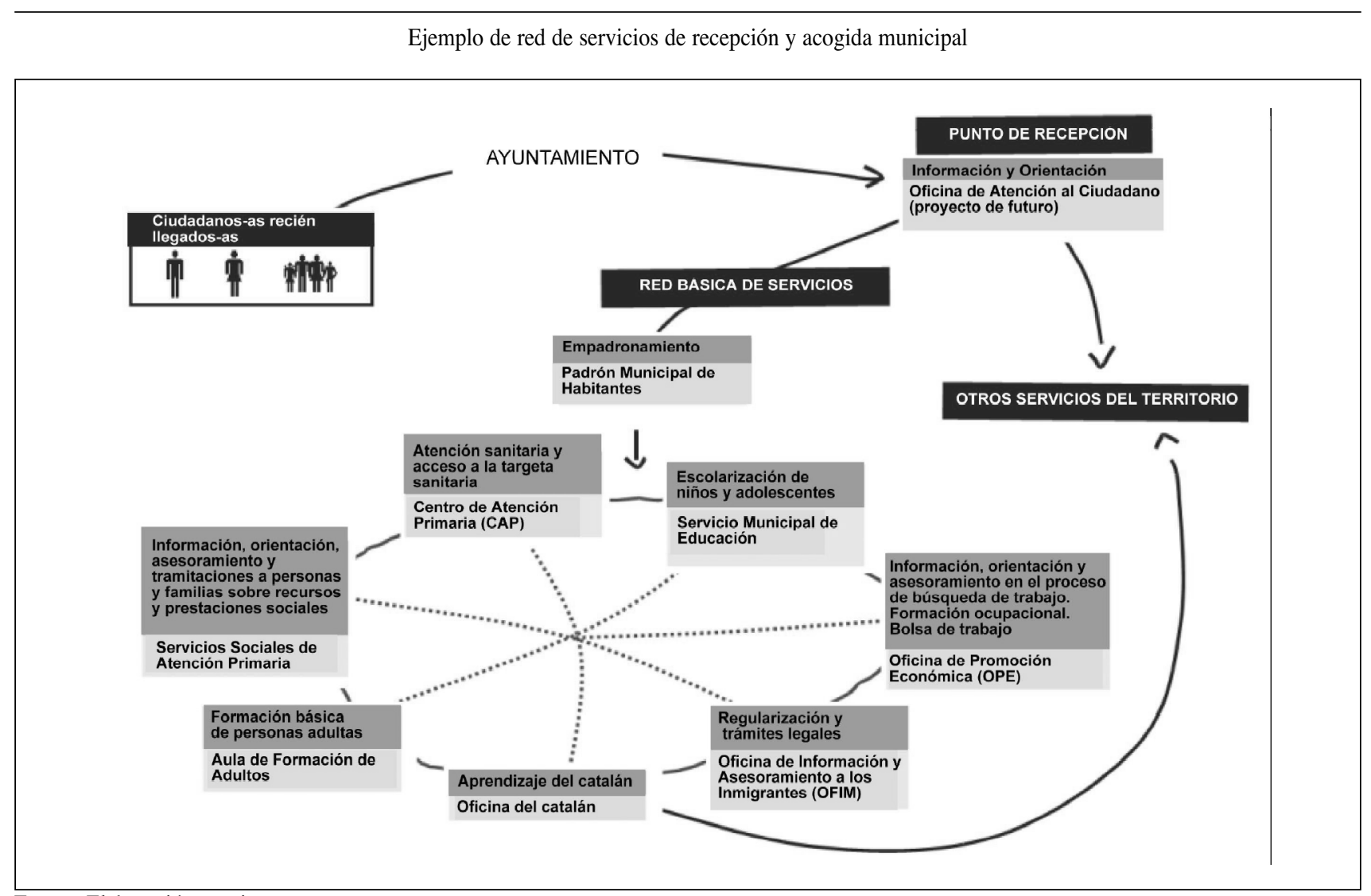

Fuente: Elaboración propia.

Los Ayuntamientos que han acogido población inmigrada en los últimos años, han desarrollado políticas y dispositivos de primera acogida; en la província de Barcelona se ha producido un trabajo importantísimo en relación al desarrollo de servicios, acciones y planes de recepción y acogida municipales; y en este último caso, la Diputación de Barcelona ha jugado un papel crucial con los Ayuntamientos para la consolidación de estos dispositivos.

Los planes locales de acogida son los instrumen- sinergias que favorecen la cohesión social en el territorio.

Observando en perspectiva el trabajo realizado desde los municipios en los últimos años, se constata la existencia de una rica experiencia acumulada en proyectos, programas y servicios de acogida en los Ayuntamientos de Cataluña, especialmente en la província de Barcelona, en donde todos y cada uno de los municipios ha realizado alguna acción en éste ámbito, como puede verse en el mapa siguiente. 
Mapa de las acciones de acogida en la provincia de Barcelona

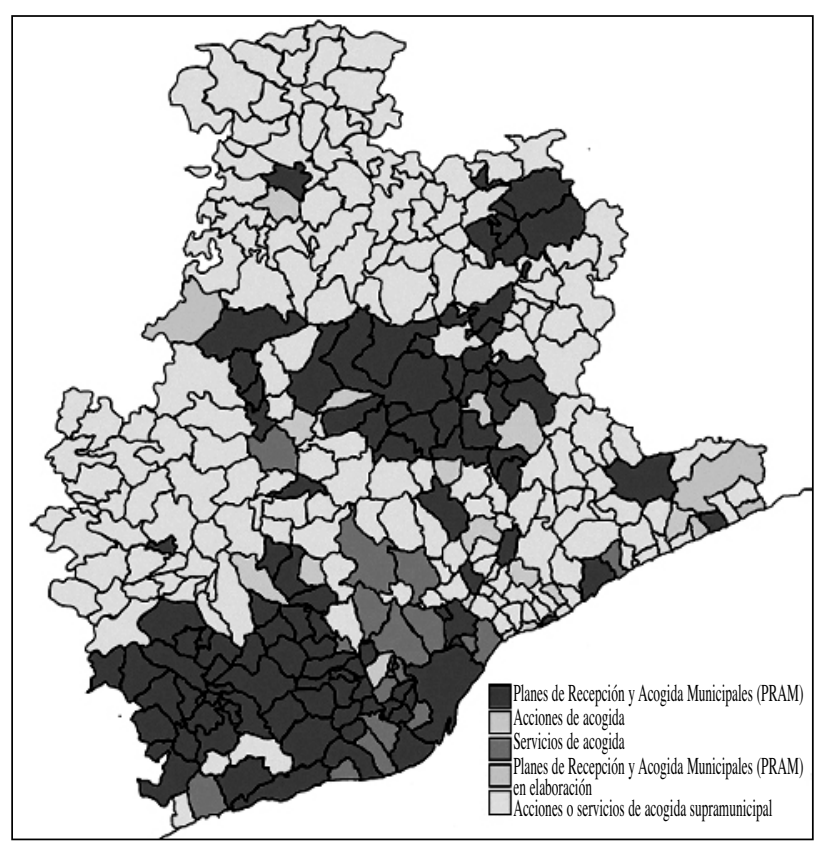

Fuente: Elaboración propia.

Planes locales de acogida: características

Los planes locales de acogida articulan sus actuaciones en base a un concepto activo de ciudadanía, alejándose de los planteamientos asistencialistas que aún perduran en la política social de nuestro país y en los que, a menudo, corren el riesgo de caer las políticas de gestión de la inmigración.

El plan local de agogida debe permitir a las personas inmigradas que adquieran progresivamente la autonomía suficiente para relacionarse con el entorno y hacerlo en igualdad de oportunidades en la sociedad receptora.

Los objetivos del plan de acogida son:

- Garantizar el acceso a la información básica de todas las personas recién llegadas, y orientarlas en los circuitos de acogida para que puedan desarrollarse con autonomía en la ciudad. Conseguir que todas las personas que llegan a la ciudad accedan a la información y a los itinerarios de acogida.

- Garantizar el acceso y uso normalizado a los servicios de acompañamiento básicos (empadronamiento, asesoramiento jurídico, sistema sanitario, servicios sociales, sistema educativo, conocimiento del entorno, aprendizaje de la lengua, asesoramiento laboral).

- Fomentar la coordinación entre los agentes sociales e institucionales que trabajan en el ámbito de la acogida a la población inmigrada.

Promover la implicación y la coresponsabilización de los agentes sociales y del conjunto de la ciudadanía en la acogida de la población recién llegada.
El plan de acogida pretende incidir sobre las principales necesidades que aparecen en el momento de la llegada y la primera instalación en el municipio; en este sentido, se estructura en base a cinco ejes estratégicos: los dos primeros inciden directamente sobre la población recién llegada, y los dos últimos lo hacen sobre los agentes de acogida:

- Información básica para la población recién llegada

- Acceso y normalización en el uso de los servicios y recursos básicos por parte de la población recién llegada

- Coordinación entre los agentes de acogida

- Planificación de los procesos de acogida

Del mismo modo, se trabaja con los siguientes ámbitos de gestión municipal, vinculados directamente con los servicios de atención y promoción de las personas: recepción o primera acogida, socio-educativo; lingüístico y laboral; sanitario; de los servicios sociales, y vivienda.

Los planes locales de acogida acostumbran a adoptar el formato de plan estratégico y, como tales, a seguir un proceso de elaboración basado en los conceptos de transversalidad, liderazgo político y técnico, participación y comunicación ciudadana.

En este sentido, hay que tener presente que:

- Son liderados por los ayuntamientos a partir de un proceso de trabajo en el que se implican también servicios dependientes de otras administraciones, entidades del territorio, así como la ciudadanía.

- Se aplican de forma temporalizada, en base a las fases de diagnóstico / análisis de la realidad, diseño / planificación, ejecución y evaluación.

- Estructuran espacios de trabajo transversal para su elaboración (o bién se adaptan a estructuras ya existentes), en el ámbito político, técnico y ciudadano, creando normalmente comisiones sectoriales.

Estos elementos metodológicos explican la voluntad de los ayuntamientos para desarrollar un trabajo de calidad, coherente tanto con las necesidades del municipio como con las posibilidades de generar respuestas y soluciones desde el territorio.

Los ayuntamientos tienen que ser, por medio de sus planes locales de acogida, las administraciones precursoras y coordinadoras de las actuaciones de acogida en su municipio.

\section{Garantizar la calidad de vida de la ciudadanía}

Éste es el segundo reto para los gobiernos locales, y para ello hace falta desarrollar políticas que favorezcan entornos físicos, urbanos, sociales, económicos y culturales de calidad.

En los últimos años, algunos núcleos antiguos de las ciudades se han convertido en territorios receptores de una parte importante de la población inmigrante. Por ello, desde las administraciones locales debemos trabajar desde las políticas de urbanismo para tener barrios de calidad, mejorar las viviendas, 
los espacios públicos... Pero estas acciones tienen que ir acompañadas de políticas de ciudadanía, imprescindibles para el progreso colectivo y la cohesión social.

En el caso de Cataluña, en los últimos años el Gobierno de la Generalitat ha puesto en marcha la Ley de barrios, que ha permitido a muchos Ayuntamientos disponer de recursos para hacer esta intervención integral en sus barrios desde el punto de vista urbanístico, pero también social.

Hay que plantear en los barrios intervenciones integrales y de consenso, que generen oportunidades y progreso colectivo, y que permitan a los ciudadanos/as no perder el sentido de identidad del barrio.

\section{Integración, convivencia y pedagogía}

El tercer reto para los gobiernos locales es la integración, la convivencia y la pedagogía, que tienen que desarrrollar para afrontar con tranquilidad el cambio social que supone la inmigración para prevenir situaciones de conflicto.

En este sentido, los programas que tanto el Área de Igualdad y Ciudadanía de la Diputación como los Ayuntamientos hemos desarrollado tienen que ver con la mediación ciudadana, la promoción del civismo, el buen uso de los espacios públicos, la promoción del asociacionismo en los barrios, la legalidad en relación a la actividad comercial, estrategias de ubicación de los equipamientos religiosos...

En relación al programa de mediación ciudadana, se configura como una estrategia útil para dar respuesta a las necesidades sociales de nuevas formas de resolución de conflictos. Desde la Diputación de Barcelona hemos puesto en marcha en 30 Ayuntamientos servicios de mediación ciudadana, creando una red de cerca de 80 mediadores/as ciudadanos/as, a quienes, desde nuestra institución, formamos y hacemos el seguimiento de su trabajo.

Desde la Diputación también trabajamos con los Ayuntamientos para el desarrollo de planes de civismo, formación, exposiciones, programas de televisión, elaboración de ordenanzas cívicas, entre otros, con el objetivo de fomentar la educación cívica.
Pero para afrontar este reto, también es muy importante apoyar el trabajo que realizan las entidades en las ciudades, sobre todo el de aquellas pequeñas entidades (asociaciones de padres y madres, de vecinos, culturales, juveniles, deportivas...) que trabajan en el barrio y que son un elemento claro para tejer la convivencia. Por ello, en la Diputación de Barcelona hemos destinado un fondo a microproyectos que desarrollan las entidades en los barrios, un apoyo que nunca puede exceder de los $3000 €$, pero que las ayuda a poner en marcha estos proyectos de convivencia allí donde realmente se necesitan, en el barrio. Pero también, en los últimos años el tejido de entidades de inmigrantes ha crecido con gran rapidez, el fortalecimiento de este asociacionismo también es importante y por ello desde la Dipuación de Barcelona hemos pactado con la Obra Social de Caixa Catalunya un fondo para apoyar a estas entidades.

Los Ayuntamientos también tienen que desarrollar programas que tengan como objetivo central trabajar especialmente con los hijos e hijas de la inmigración. Con el objetivo de conseguir la integración y la participación de los jóvenes en la vida social, y huir de situaciones como las que se produjeron en algunos barrios de las ciudades francesas en el año 2005.

\section{Cantidad y calidad de los servicios públicos}

Aún hay un cuarto reto para los gobiernos locales, que es la cantidad y la calidad de los servicios públicos. La presencia de la población inmigrada en determinadas áreas urbanas cercana al 30 o al $40 \%$ supone un incremento de la demanda y el consumo colectivo de servicios públicos, y no siempre conlleva un incremento de los recurosos, lo cual afecta directamente a la calidad de estos servicios.

Esta situación produce a la ciudadanía una percepción de pérdida de calidad de vida por la llegada de nueva población, y el consiguiente rechazo e incertidumbre hacia la inmigración. Esta nueva realidad también implica que algunos Ayuntamientos hayan puesto en marcha observatorios de calidad de los servicios públicos, con la intención de obtener datos sobre la evolución de las necesi- 
dades de servicios públicos y de coordinarse entre todas las administraciones que los ofrecen para mejorar los servicios que se prestan al conjunto de la ciudadanía.

\section{Las políticas de ciudadanía}

Llegados a este punto, ya podemos afirmar que la gestión de la inmigración ha puesto de relieve y en parte en crisis muchos de los conceptos que tradicionalmente se han utilizado en la definición de las políticas públicas. En la gestión de la inmigración, en un primer momento, se partió de la idea que el sujeto de la acción pública era el inmigrante y por tanto se desarrollaron programas dirigidos a este segmento de la población. Pero este primer estadio, en poco tiempo, quedó superado con la formulación de los conceptos de integración y acomodación, pasando a ser el sujeto de la acción pública el conjunto de la ciudadanía. En este marco es en el que la Diputación de Barcelona a través del Área de Igualdad y Ciudadanía, inscribimos nuestro trabajo para la gestión de la inmigración.

Porque el cambio social que supone la inmigración requiere nuevos enfoques, instrumentos, recursos y estrategias, requiere políticas que tengan como finalidad la capacitación de las personas, del conjunto de la ciudadanía. Y en este marco es donde se situan las políticas de ciudadanía.

\section{Breve apunte histórico}

En los años 80, en muchos Ayuntamientos se crean las áreas de servicios sociales como respuesta de la transición a la falta de políticas de bienestar en las ciudades. Estas áreas se ocupan fundamentalmente de las necesidades de las personas y de los colectivos que necesitan asistencia social. En los años 90 empiezan a aparecer las áreas de servicios personales, que suponen una redefinición y ampliación de los organigramas municipales, integrando ámbitos de acción que identifican zonas emergentes de intervención: juventud, tercera edad, mujer, participación, cultura, educación, deportes, solidaridad...
Estas políticas parten del concepto de comunidad y de trabajo comunitario. La comunidad se entiende como "Un grupo social formado por individuos relacionados entre ellos por vínculos de diferentes tipos de los cuales surge un sentimiento de pertenencia a un grupo, y por tanto unos ciertos valores compartidos, tanto dentro del grupo en si como en el marco del territorio que habita" (Plana y Gomá, 1997).

Vemos que en los años 80 y 90 se considera la heterogeneidad como fuente de conflicto y se gestiona hacia la homogeneidad. En la actualidad, el concepto de comunidad ha quedado obsoleto para explicar las realidades cambiantes de barrios y ciudades. A nuestro entender, es más adecuado introducir el concepto de esfera pública y pensar en la ciudadanía como un proceso de construcción a lo largo de la vida.

Nos encontramos ante un nuevo escenario en donde conviven las políticas que se han desarrollado desde los servicios sociales, es decir de soporte a las necesidades de las personas, y las políticas de ciudadanía que dan soporte a las capacidades de las personas. Las políticas de ciudadanía y las de servicios sociales (dependencia) tienen perspectivas distintas pero estrategias complementarias.

\section{Abordan el cambio social y los conflictos fuertes}

El primer elemento para definir las políticas de igualdad y ciudadanía es que abordan el cambio social. Habitualmente, este cambio social se expresa en forma de conflicto; por ejemplo, el conflicto que se genera en el hogar por la repartición de las tareas domésticas, sería un conflicto privado, pero justo detrás se esconde un cambio social, que es la igualdad real entre hombres y mujeres.

Estos conflictos, según el profesor Ricard Zapata, se pueden clasificar en fuertes y débiles. Los conflictos débiles son aquellos que para resolverlos requieren sólo de una decisión de carácter administrativo; pongamos por ejemplo que un Ayuntamiento recibe una demanda de la ciudadanía para construir un determinado equipamiento, se trata de un conflicto que por medio de una decisión administrativa se soluciona. Previamente es necesaria una decisión 
política, pero ésta no requiere de innovación política, sólo necesita la ampliación de recursos para la provisión de este equipamiento.

En cuanto a los conflictos fuertes, estos requieren de innovación y de decisión política en forma de leyes, de medidas..., y al mismo tiempo provocan cambios estructurales en la sociedad; hablamos por ejemplo del conflicto que genera la necesidad de igualdad real de las mujeres, que requiere establecer nuevas medidas y leyes que den respuesta a esta demanda.

Nos encontramos en una época que se caracteriza por el incremento cada vez más pronunciado de los conflictos fuertes, la globalización económica, las migraciones, la diversidad... Estos son algunos de los ejemplos que la gobernabilidad tiene que abordar desde nuevas formas de actuación y decisión política porque son cada vez más protagonistas en las agendas públicas.

Así pues, a partir de esta primera descripción podemos afirmar que las políticas de igualdad y ciudadanía son políticas que abordan el cambio social y los conflictos fuertes que modifican o cuestionan las estructuras sociales vigentes.

\section{El concepto de ciudadanía}

El segundo de los elementos teóricos para la definición de las políticas de igualdad y ciudadanía es el propio concepto de ciudadanía. Las tres tradiciones de pensamiento político que han operado históricamente han sido la liberal, la comunitarista y la republicana.

El pilar básico para la tradición liberal es el estado, para la comunitarista la nacionalidad y para la republicana la esfera pública. En cuanto al contenido, para los liberales la ciudadanía son derechos (civiles, políticos, sociales...), para los comunitaristas es identidad política, lealtad de sentimientos y de pertenencia a una comunidad nacional, y para los republicanos la ciudadanía es una práctica que implica obligaciones, deberes y responsabilidades. También la tradición republicana se diferencia de la liberal y la comunitarista en que la ciudadanía es una dimensión activa, no pasiva, el ciudadano/a se hace a lo largo de su vida. En este sentido, la tradición republicana es la que nos ofrece mayores elementos de innovación para la definición de las políticas de igualdad y ciudadanía.

Así pues, en relación a este segundo elemento podemos concluir que las políticas de igualdad y ciudadanía tienen la misión de formar ciudadanas y ciudadanos activos, responsables y comprometidos con la esfera pública.

\section{Políticas proactivas}

El tercer de los elementos para definir las políticas de ciudadanía es la consideración de su estrategia principal, es decir si son preventivas (quieren evitar el conflicto), reactivas (las que reaccionan ante un conflicto, tratando de evitarlo) y las proactivas (ven el conflicto como oportunidad).

En este sentido, entendemos que las estrategias preventivas y las reactivas se aplican a las políticas de dependencia, en cambio las estrategias proactivas desarrollan las capacidades de las personas para que se acomoden a los procesos de cambio y gestionen el conflicto con autonomía. Así pues, estas últimas son más eficaces en la gestión del conflicto y en las políticas de igualdad y ciudadanía.

A partir de este tercer elemento, podemos decir que las políticas de igualdad y ciudadanía utilizan las estrategias proactivas, son políticas para capacitar a las personas y no para asistir a la dependencia.

\section{Las nuevas demandas ciudadanas}

El cuarto elemento teórico a considerar en la definición de las políticas de igualdad y ciudadanía son las nuevas demandas ciudadanas. Demandas como la defensa de los derechos jurídicos de los homosexuales, de las familias monoparentales, la conciliación de la vida personal, laboral y familiar, entre otras, expresan los conflictos y los cambios sociales que están ocurriendo en la sociedad del siglo XXI. Y las políticas de igualdad y ciudadanía deben dar respuesta a estas nuevas demandas.

Éste es el nuevo escenario, con la formulación de nuevas demandas ciudadanas que requieren, en muchos casos por parte de los poderes públicos, la 
articulación de estrategias distintas a las aplicadas hasta este momento. Un escenario que ha propiciado la elaboración y la puesta en marcha de las políticas de igualdad y ciudadanía, creando un marco de trabajo distinto al desarrollado por las áreas de servicios sociales.

\section{La organización de las políticas de igualdad y ciudadanía. El caso del Área de Igualdad y Ciudadanía de la Diputación de Barcelona}

En los Ayuntamientos y en la misma Diputación de Barcelona, a lo largo de estos años se han ensayado diversas fórmulas de trabajo para ubicar la gestión de la inmigración (dependencia de alcaldía o presidencia, comisionados, consorcios...), pero finalmente la Diputación de Barcelona optó por constituir, en julio de 2004, el Área de Igualdad y Ciudadanía, en la que se integraron un conjunto de políticas transversales, como las de juventud, igualdad de género e inmigración.

Estos tres sectores tenían en común planes transversales. El plan de juventud se elaboró como una herramienta de coordinación de las actuaciones que se estaban delegando en otras áreas de la Diputación. El plan de Igualdad surgió de las recomendaciones europeas de la transversalidad de género. Y el plan de inmigración y diversidad se realizó con la idea de analizar y explicar cómo la inmigración impactaba en el conjunto de los servicios públicos.

Los tres sectores, juventud, igualdad de género e inmigración utilizaban la transversalidad como metodología de trabajo, habitual en las políticas de igualdad y ciudadanía, dado que la complejidad de los cambios sociales sobre los que operamos en estas políticas obliga a la coordinación de muchas actuaciones que desarrollan otras partes de la organización. En este último mandato hemos incluido también en este área de gobierno las políticas de participación ciudadana.

El Área de Igualdad y Ciudadanía tiene la misión de liderar la acción pública relacionada con la igualdad de oportunidades, la cohesión social y la promoción del concepto de ciudadanía.

Y por ello, en el Plan de Mandato para el 20072011 de la Diputación de Barcelona, una de las bases de gobierno es la relacionada con las acciones en favor de la convivencia y la ciudadanía: «Desde la Diputación de Barcelona queremos liderar una nueva acción pública que ponga el acento en la capacitación ciudadana. Se trata de impulsar un conjunto de políticas con el objetivo de hacer más capaces y responsables a nuestros ciudadanos y ciudadanas. Son políticas fundamentadas en el hecho de que la ciudadanía es una construcción activa, que comporta derechos y deberes mutuos, que se construye de forma individual a lo largo de toda la vida y que tiene una voluntad transformadora.

En este ámbito se incluyen las políticas de igualdad de género y las políticas de acogida, integración y mediación ciudadana, que quieren responder a los retos del fenomeno migratorio. Del mismo modo, desarrollaremos políticas para reforzar la ciudadanía activa de los jóvenes y su emancipación, así como la autonomía y la implicación de las personas mayores.

No menos importantes son las políticas de promoción de la participación ciudadana. Nos comprometemos a trabajar con los municipios para fortalecer la dimensión cívica, ciudadana y democrática de nuestros pueblos y ciudades.» (Diputación de Barcelona, 2008).

Con este objetivo, realizamos programas y acciones transversales, con la voluntad de capacitar a toda la ciudadanía para que pueda desarrollarse de manera autónoma y en igualdad de oportunidades. Programas que destinamos a los Ayuntamientos y entes locales, con quienes trabajamos en red para llegar a todas las personas. Programas como el de mediación ciudadana, ya mencionado, como los agentes locales de diversidad, igualdad y juventud, nuevos perfiles profesionales al servicio de las políticas de ciudadanía en los Ayuntamientos. Pero también acciones que destinamos directamente a la ciudadanía, como las ayudas a entidades sociales que trabajan en el territorio o el soporte para desarrollar proyectos de convivencia a entidades formadas por personas recién llegadas, en convenio con Caixa Catalunya.

Son sólo algunos ejemplos de políticas de ciudadanía que desarrollamos desde el Área de Igualdad y Ciudadanía de la Diputación de Barcelona con el convencimiento de que la gestión 
de la inmigración pide trabajar con todas las personas para fomentar la convivencia ciudadana y la cohesión social. Porque nuestra obligación como administración pública de proximidad es estar al lado de la ciudadanía, responder a sus necesidades y demandas. Y ahora, nuestros conciudadanos y conciudadanas nos piden entender la nueva configuración social. Y esto sólo puede conseguirse desde el conocimiento mútuo: las personas recién llegadas tienen que conocer la sociedad de acogida, y los autóctonos, las nuevas culturas que enriquecen los barrios. Para así fomentar un nuevo concepto de pertinencia basado en la diversidad.

Ésta es nuestra filosofía de trabajo, la del Área de Igualdad y Ciudadanía de la Diputación de Barcelona.

\section{Conclusiones}

Las políticas de igualdad y ciudadanía se caracterizan por:

- Abordar el cambio social y los conflictos fuertes que modifican o cuestionan las estructuras sociales vigentes.

- Formar ciudadanas y ciudadanos activos, responsables y comprometidos con la esfera pública.

- Capacitar a las personas.

- Dar respuesta a las nuevas demandas ciudadanas.

- Utilizar las estrategias proactivas, de acompañamiento de la ciudadanía, de capacitación, de transformación social.

Por ello, desde nuestra perspectiva, situamos la gestión de la inmigración en este ámbito, ya que entendemos que debemos actuar sobre el conjunto de la ciudadanía para afrontar con tranquilidad el cambio social que supone la inmigración.

Para gestionar con éxito las políticas de inmigración, debemos abordar el tema desde una pers- pectiva global, no estrictamente desde los servicios sociales, es necesaria la implicación de las distintas áreas del Ayuntamiento (educación, cultura, urbanismo, oficina de atención al ciudadano, salud...); requiere pues de un trabajo transversal y de estrategias compartidas por los distintos servicios municipales.

Para gestionar con éxito las políticas de inmigración es necesario el trabajo en red, es decir contando con la complicidad de las entidades que operan en el territorio (Cáritas, Cruz Roja, CITE, AMIC, entidades vecinales...). En este sentido también es muy útil fortalecer la actividad de estas entidades y especialmente de aquellas que están trabajando en los barrios, y que más directamente pueden favorecer la convivencia en los espacios más cercanos a la ciudadanía (la escuela, la escalera de vecinos...).

Para gestionar con éxito las políticas de inmigración, debemos pensar en el conjunto de la ciudadanía y para ello establecer acciones, programas y estrategias que van más allá de la recepción y la acogida (mediación, civismo, convivencia,...).

Cualquier organización puede ser buena siempre que respete estos principios y el enfoque que planteamos para afrontar la gestión de la inmigración y el cambio social que conlleva.

\section{Referencias}

Diputación de Barcelona, Servicio de Políticas de Diversidad y Ciudadanía del Área de Igualdad y Ciudadanía (2004). Elementos para una actuación municipal de recepción y acogida de inmigrantes.

Diputación de Barcelona (2008). Plan de Actuación del Mandato 2008-2011.

Plana, J. y Gomà, R. (1997). Revista CIFA.

Manuscrito recibido: 26/01/2009

Revisión recibida: 15/03/2009

Manuscrito aceptado: 13/04/2009 